\title{
Universal Borromean Binding in Spin-Orbit-Coupled Ultracold Fermi Gases
}

\author{
Xiaoling Cui ${ }^{1, *}$ and Wei $\mathrm{Yi}^{2,3, \dagger}$ \\ ${ }^{1}$ Beijing National Laboratory for Condensed Matter Physics, Institute of Physics, \\ Chinese Academy of Sciences, Beijing 100190, People's Republic of China \\ ${ }^{2}$ Key Laboratory of Quantum Information, University of Science and Technology of China, \\ CAS, Hefei, Anhui 230026, People's Republic of China \\ ${ }^{3}$ Synergetic Innovation Center of Quantum Information and Quantum Physics, University of Science \\ and Technology of China, Hefei, Anhui 230026, People's Republic of China \\ (Received 31 March 2014; revised manuscript received 11 June 2014; published 13 August 2014)
}

\begin{abstract}
Borromean rings and Borromean binding, a class of intriguing phenomena as three objects are linked (bound) together while any two of them are unlinked (unbound), widely exist in nature and have been found in systems of biology, chemistry, and physics. Previous studies have suggested that the occurrence of such a binding in physical systems typically relies on the microscopic details of pairwise interaction potentials at short range and is, therefore, nonuniversal. Here, we report a new type of Borromean binding in ultracold Fermi gases with Rashba spin-orbit coupling, which is universal against short-range interaction details, with its binding energy only dependent on the $s$-wave scattering length and the spin-orbit-coupling strength. We show that the occurrence of this universal Borromean binding is facilitated by the symmetry of the single-particle dispersion under spin-orbit coupling and is, therefore, symmetry selective rather than interaction selective. The state is robust over a wide range of mass ratios between composing fermions, which are accessible by $\mathrm{Li}-\mathrm{Li}, \mathrm{K}-\mathrm{K}$, and K-Li mixtures in cold-atom experiments. Our results reveal the importance of single- particle spectral symmetry in few-body physics and shed light on the emergence of new quantum phases in a many-body system with exotic few-body correlations.
\end{abstract}

DOI: 10.1103/PhysRevX.4.031026

\section{INTRODUCTION}

The fascinating topological structure of Borromean rings has attracted much attention in biology [1] and chemistry [2], while, in physics, their quantum mechanical analog, the Borromean binding, has been reported in halo nuclei ${ }^{6} \mathrm{He}$ and ${ }^{11} \mathrm{Li}[3,4]$ and in ultracold atomic gases [5-14] manifested as the Efimov effect $[15,16]$. Despite its wide existence in nature, the Borromean phenomenon seems quite intricate and peculiar, as it especially requires three bodies being more favorably bound than two bodies. Previous studies have shown that such a requirement can be fulfilled by fine-tuning the pairwise short-range interaction potentials. For instance, in three dimensions (3D), the coupling constant should vary with the specific shape of the short-range potential $[17,18]$, while in two dimensions (2D), it is necessary for the potential to include a repulsive barrier outside an attractive core [19-21]. Meanwhile, for Efimov-type Borromean states, a short-range (three-body) parameter is essential to uniquely determine the binding

\footnotetext{
*xlcui@iphy.ac.cn

†wyiz@ustc.edu.cn
}

Published by the American Physical Society under the terms of the Creative Commons Attribution 3.0 License. Further distribution of this work must maintain attribution to the author(s) and the published article's title, journal citation, and DOI.
Subject Areas: Atomic and Molecular Physics

energies as well as the locations of their emergence [16]. In all these studies, the Borromean binding appears to be a nonuniversal phenomenon that inevitably relies on the short-range details of interaction potentials. This nonuniversality makes a unified understanding of the Borromean binding conceptually difficult and renders its experimental detection inconveniently system dependent.

To overcome these difficulties, we aim at engineering a universal Borromean binding, where the short-range interaction details are completely irrelevant and its occurrence is physically transparent. Motivated by a simple fact that fewbody physics also crucially depends on single-particle properties, we realize that a potential route toward our goal is through the modification of single-particle physics. In ultracold atomic gases, an outstanding candidate to achieve this modification is the synthetic spin-orbit coupling (SOC) [22-32], with the form of SOC highly tunable according to a number of proposals [33-39]. Indeed, the significant change of single-particle dispersion by SOC has been shown to result in rich and exciting physics in few- and many-body systems [40]. In particular, it has been found that an isotropic SOC can support a dimer for arbitrarily weak interactions [39,41-43] and can induce a universal trimer in a wide parameter regime of interaction strength and mass ratio [44]. These states are in distinct contrast to the dimer and the Kartavtsev-Malykh trimer [45] 
in the absence of SOC. So far, however, no universal Borromean binding has yet been identified.

In this work, we report the discovery of universal Borromean bindings in ultracold Fermi-Fermi mixtures with Rashba SOC. The three-body system can be denoted as $\tilde{a}-\tilde{a}-b$, where $\tilde{a}$ is a two-component fermion subject to Rashba SOC, with one of its components tuned close to a wide Feshbach resonance with the $b$ atom [46]. The mechanism for the Borromean binding in this system is schematically shown in Fig. 1. Under Rashba SOC, the single-particle ground state of $\tilde{a}$ possesses a $\mathrm{U}(1)$ degeneracy [see Fig. 1(a)]. With such a spectral symmetry, the two-body $(\tilde{a}-b)$ scattering within the lowest-energy subspace is blocked due to total momentum conservation [Fig. 1(b)], which effectively suppresses the dimer formation. In contrast, the three-body scattering can take full advantage of this U(1) degeneracy, where an initial state of $\tilde{a}-\tilde{a}-b$ atoms at $\{\mathbf{k},-\mathbf{k}, 0\}$ can be scattered to a different state at $\left\{\mathbf{k}^{\prime},-\mathbf{k}^{\prime}, 0\right\}$ with a conserved total momentum [Fig. 1(c)]. Here, $\mathbf{k}$ and $\mathbf{k}^{\prime}$ both lie on the circle of the U(1) degenerate manifold of $\tilde{a}$. This enhanced low-energy scattering phase space strongly suggests that the trimer formation is much easier than the dimer formation, which, as we will show, would give rise to the Borromean binding. As the emergence of this Borromean binding is symmetry selective rather than interaction selective, its universality is naturally guaranteed: The binding energy only relies on the

(a)

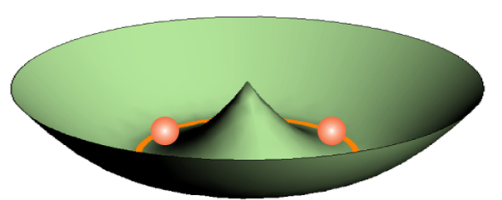

(b)

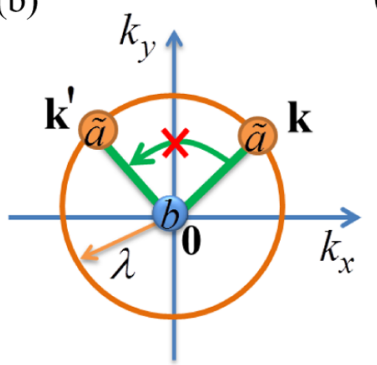

(c)

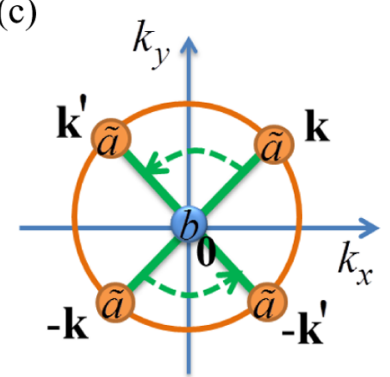

FIG. 1. Illustration of the Borromean binding mechanism in the $\tilde{a}-\tilde{a}-b$ system. (a) Under Rashba SOC, the single-particle ground state of $\tilde{a}$ has a $\mathrm{U}(1)$ degeneracy in the $\left(k_{x}, k_{y}\right)$ plane with radius $k_{\perp}=\lambda$. (b) The two-body $\tilde{a}-b$ system cannot scatter within the lowest-energy subspace due to the conservation of total momentum. (c) In contrast, the scattering of the three-body $\tilde{a}-\tilde{a}-b$ system is allowed within the lowest-energy subspace through virtual scattering to states like $\left\{\mathbf{k}^{\prime},-\mathbf{k}, \mathbf{k}-\mathbf{k}^{\prime}\right\}$ or $\left\{\mathbf{k},-\mathbf{k}^{\prime}, \mathbf{k}^{\prime}-\mathbf{k}\right\}$ (dashed green arrows). The dramatic enhancement of the low-energy scattering phase space in (c) gives rise to the Borromean binding. $s$-wave scattering length and the SOC strength. We identify the existence of such bindings in a wide range of mass ratios between composing fermions, which are readily accessible by $\mathrm{Li}-\mathrm{Li}, \mathrm{K}-\mathrm{K}$, and $\mathrm{K}-\mathrm{Li}$ mixtures in current cold-atom experiments. The robustness of this Borromean binding suggests the importance of the single-particle spectral symmetry in few-body physics, which has rarely been discussed before.

\section{MODEL}

The Hamiltonian of our system is written as

$$
\begin{aligned}
H= & \sum_{\mathbf{k}, \alpha=\uparrow, \downarrow} \frac{\mathbf{k}^{2}}{2 m_{a}} a_{\mathbf{k}, \alpha}^{\dagger} a_{\mathbf{k}, \alpha}+\sum_{\mathbf{k}} \frac{\mathbf{k}^{2}}{2 m_{b}} b_{\mathbf{k}}^{\dagger} b_{\mathbf{k}} \\
& +\frac{\lambda}{m_{a}} \sum_{\mathbf{k}}\left[\left(k_{x}-i k_{y}\right) a_{\mathbf{k}, \uparrow}^{\dagger} a_{\mathbf{k}, \downarrow}+\text { H.c. }\right] \\
& +\frac{U}{V} \sum_{\mathbf{k}, \mathbf{k}^{\prime}, \mathbf{Q}} a_{\mathbf{k}, \uparrow}^{\dagger} b_{\mathbf{Q}-\mathbf{k}}^{\dagger} b_{\mathbf{Q}-\mathbf{k}^{\prime}} a_{\mathbf{k}^{\prime}, \uparrow},
\end{aligned}
$$

where $\lambda$ is the strength of the Rashba SOC between two spin species $(\alpha=\uparrow, \downarrow)$ of the $\tilde{a}$ atom; $U$ is the bare interaction between $a_{\uparrow}$ and $b$ and is related to the $s$-wave scattering length $a_{s}$ via $1 / U=\mu /\left(2 \pi a_{s}\right)-(1 / V) \sum_{\mathbf{k}} 1 /$ $\left(2 \mu \mathbf{k}^{2}\right)$, with $V$ the quantization volume and $\mu=$ $m_{a} m_{b} /\left(m_{a}+m_{b}\right)$ the reduced mass. As Feshbach resonances are state dependent and have a finite width, it is reasonable to assume negligible interactions in other twobody subsystems [46]. Note that we have taken $\hbar=1$ for brevity.

Under SOC, the single-particle eigenstate of $\tilde{a}$ in the helicity basis is created by $a_{\mathbf{k}, \sigma}^{\dagger}=\sum_{\alpha} \gamma_{\mathbf{k}, \sigma}^{\alpha} a_{\mathbf{k}, \alpha}^{\dagger}$, where $\sigma= \pm, \gamma_{\mathbf{k}, \pm}^{\uparrow}= \pm e^{ \pm i \phi_{k} / 2} / \sqrt{2}, \gamma_{\mathbf{k}, \pm}^{\downarrow}=e^{ \pm i \phi_{k} / 2} / \sqrt{2}$, and $\phi_{k}=$ $\arg \left(k_{x}, k_{y}\right)$. The corresponding eigenenergy is $\epsilon_{\mathbf{k}, \sigma}^{a}=$ $\left[\left(k_{\perp}+\sigma \lambda\right)^{2}+k_{z}^{2}\right] /\left(2 m_{a}\right)+E_{\mathrm{th}}$, with $k_{\perp}=\sqrt{k_{x}^{2}+k_{y}^{2}}$. The ground state has $\mathrm{U}(1)$ degeneracy in $\mathbf{k}$ space with $k_{\perp}=\lambda$ and a threshold energy $E_{\mathrm{th}}=-\lambda^{2} /\left(2 m_{a}\right)$. Given the singleparticle spectrum $\epsilon_{\mathbf{k}}^{b}=\mathbf{k}^{2} /\left(2 m_{b}\right)$ for the $b$ atom, the twobody $\tilde{a}-b$ and the three-body $\tilde{a}-\tilde{a}-b$ systems, respectively, have threshold energies $E_{\mathrm{th}}$ and $2 E_{\mathrm{th}}$.

\section{DIMER STATE}

We start by addressing the dimer state of the $\tilde{a}$ - $b$ system. The dimer wave function with a center-of-mass momentum $\mathbf{Q}$ can be written as

$$
\left|\Psi^{(2)}\right\rangle=\sum_{\mathbf{k}, \sigma= \pm} \Psi^{(2)}(\mathbf{Q}-\mathbf{k} ; \mathbf{k} \sigma) b_{\mathbf{Q}-\mathbf{k}}^{\dagger} a_{\mathbf{k} \sigma}^{\dagger}|0\rangle .
$$

The coefficient $\Psi^{(2)}$ can be solved in a standard way based on the Lippmann-Schwinger equation [47]: 


$$
\Psi^{(2)}(\mathbf{Q}-\mathbf{k} ; \mathbf{k} \sigma) \propto \frac{\gamma_{\mathbf{k} \sigma}^{\uparrow}{ }^{*}}{E_{2}+E_{\mathrm{th}}-\epsilon_{\mathbf{Q}-\mathbf{k}}^{b}-\epsilon_{\mathbf{k}, \sigma}^{a}},
$$

where $E_{2}$ is the two-body binding energy determined by

$$
\frac{1}{U}=\frac{1}{V} \sum_{\mathbf{k}, \sigma} \frac{\left|\gamma_{\mathbf{k} \sigma}^{\uparrow}\right|^{2}}{E_{2}+E_{\mathrm{th}}-\epsilon_{\mathbf{Q}-\mathbf{k}}^{b}-\epsilon_{\mathbf{k}, \sigma}^{a}} .
$$

Among all $\mathbf{Q}$ sectors, the lowest bound state $\left(E_{2}<0\right)$ is found with $\mathbf{Q}=0$. Different from previous two-body solutions with Rashba SOC [41], to support a bound state here, the interaction strength $1 /\left(\lambda a_{s}\right)$ must be greater than a finite critical value $1 /\left(\lambda a_{s}\right)_{c}$, which can be solved analytically as a function of mass ratio $\eta=m_{a} / m_{b}$ :

$$
\frac{1}{\left(\lambda a_{s}\right)_{c}}=x\left(1-\frac{x}{2} \ln \frac{1+x}{1-x}\right), \quad x=\frac{1}{1+\eta} .
$$

The function of $1 /\left(\lambda a_{s}\right)_{c}$ in terms of $\eta$ is plotted in Fig. 2(a). As $\eta$ is increased from $0,1 /\left(\lambda a_{s}\right)_{c}$ first increases from $-\infty$ to a positive maximum value around $\eta \sim 1$, then decreases and finally approaches $0^{+}$as $\eta \rightarrow \infty$. This behavior can be understood from the analysis of the two-body scattering energy $E_{\mathbf{k}, \sigma}^{(2)}=\epsilon_{-\mathbf{k}}^{b}+\epsilon_{\mathbf{k}, \sigma}^{a}-E_{\mathrm{th}}$, whose low-energy property is crucial for the formation

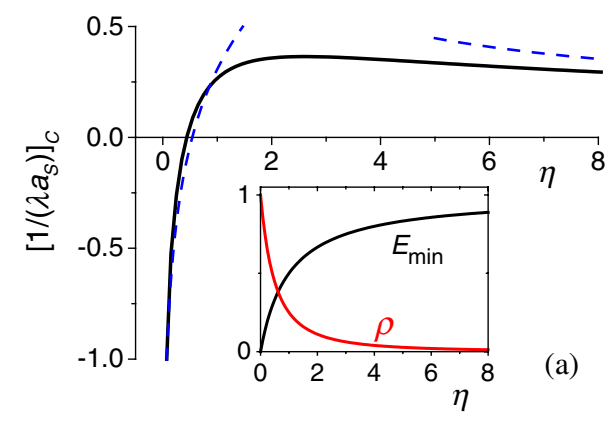

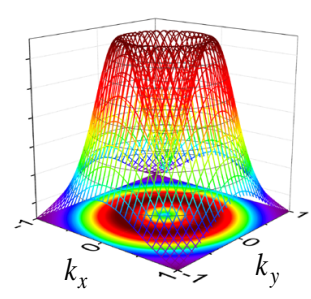

(b1)

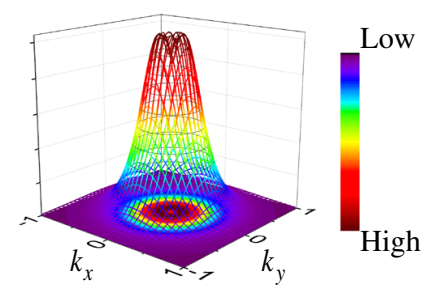

(b2)
FIG. 2. Dimer threshold and momentum distribution. (a) Critical interaction strength $1 /\left(\lambda a_{s}\right)_{c}$ to support a bound state of an $\tilde{a}-b$ system as a function of the mass ratio $\eta=m_{a} / m_{b}$. Dashed lines show the asymptotic fits $1-1 / 2 \ln (4 / \eta)$ and $\eta^{-1 / 2}$, respectively, in the limits of $\eta \rightarrow 0$ and $\infty$. The insets show the minimum of the two-body scattering energy $E_{\min }$ [in units of $\left.\lambda^{2} /\left(2 m_{a}\right)\right]$ and the density of states $\rho$ (in units of $4 \pi^{2} m_{a} \lambda$ ) at $E_{\min }$. (b1),(b2) Probability distribution of a shallow dimer in the $\left(k_{x}, k_{y}\right)$ plane $\left|\Psi^{(2)}(-\mathbf{k} ; \mathbf{k},-)\right|^{2}$ for two different mass ratios $\eta=1$ and $40 / 6$, respectively, at $1 /\left(\lambda a_{s}\right)=0.3,0.35$. of a shallow bound state. It is easy to see that the minimum of $E_{\mathbf{k}, \sigma}^{(2)}$, denoted as $E_{\min }$, lies on a ring with radius $k_{\perp}=\lambda /(1+\eta)$ in the $\left(k_{x}, k_{y}\right)$ plane. As $\eta$ increases from 0 to $\infty$, the radius evolves from $\lambda$ to 0 , indicating a dimensional crossover from effectively $2 \mathrm{D}$ to $3 \mathrm{D}$. This effective dimensional crossover is also manifested in the density of states $\rho$ at $E_{\min }$, which approaches 0 from a finite value as $\eta$ increases [see the inset of Fig. 2(a)]. Consequently, the critical $1 /\left(\lambda a_{s}\right)_{c}$ changes from $-\infty$ to 0 , corresponding to an effective dimensional crossover from 2D to 3D without SOC.

An important feature in Fig. 2(a) is that the two-body threshold $1 /\left(a_{s}\right)_{c}$ is pushed from resonance to positive values for a considerable range of mass ratio $\eta \in[0.44, \infty)$, indicating the suppression of dimer formation by Rashba SOC. The suppression of dimer is consistent with the schematic picture in Fig. 1(b). For an initial $\tilde{a}-b$ state in the lowest-energy subspace $(|\mathbf{Q}|=\lambda)$, it cannot be scattered into a different state among the $\mathrm{U}(1)$ degenerate ground states due to the conservation of total momentum. Given the blocked threshold scattering with $|\mathbf{Q}|=\lambda$, the groundstate dimer with $E_{2}<0$ is found to be at $\mathbf{Q}=0$, where the $\mathrm{U}(1)$ symmetry is restored at the cost of higher threshold energy $\left(E_{\min }>0\right)$. In Figs. 2(b1) and 2(b2), we plot the momentum distribution of such dimers for two different mass ratios $\eta=1$ and $40 / 6$, corresponding to the cases of $\mathrm{Li}-\mathrm{Li}$ (or $\mathrm{K}-\mathrm{K}$ ) and $\mathrm{K}-\mathrm{Li}$ mixtures. For both cases, the largest weight of the wave function lies on a ring with radius $k_{\perp}<\lambda$ and with $E_{\min }>0$.

\section{BORROMEAN BINDING}

We are now in position to examine the threebody problem. According to the analysis in Fig. 1(c), the ground-state trimer is expected to have zero center-ofmass momentum, for which the wave function can be written as

$$
\left|\Psi^{(3)}\right\rangle=\sum_{\mathbf{k} \sigma} \sum_{\mathbf{q} \xi} \Psi^{(3)}(-\mathbf{k}-\mathbf{q} ; \mathbf{k} \sigma ; \mathbf{q} \xi) b_{-\mathbf{k}-\mathbf{q}}^{\dagger} a_{\mathbf{k} \sigma}^{\dagger} a_{\mathbf{q} \xi}^{\dagger}|0\rangle .
$$

Following similar procedures as in solving the two-body problem, we obtain the integral equations for the threebody bound-state solution [47]:

$$
\frac{1}{U} F_{\sigma}(\mathbf{k})=\frac{1}{V} \sum_{\mathbf{q} \xi} \frac{\left|\gamma_{\mathbf{q} \xi}^{\uparrow}\right|^{2} F_{\sigma}(\mathbf{k})-\left|\gamma_{\mathbf{k} \sigma}^{\uparrow}\right|^{2} F_{\xi}(\mathbf{q})}{E_{3}+2 E_{\mathrm{th}}-\epsilon_{-\mathbf{k}-\mathbf{q}}^{b}-\epsilon_{\mathbf{k}, \sigma}^{a}-\epsilon_{\mathbf{q}, \xi}^{a}},
$$

where $F_{\sigma}(\mathbf{k})=U \sum_{\mathbf{q} \xi} \Psi^{(3)}(-\mathbf{k}-\mathbf{q} ; \mathbf{k} \sigma ; \mathbf{q} \xi) \gamma_{\mathbf{k} \sigma}^{\uparrow} \gamma_{\mathbf{q} \xi}^{\uparrow}$, and the trimer binding energy $E_{3}$ can be obtained by requiring a nonzero solution of $F_{\sigma}(\mathbf{k})$. Under Rashba SOC, the $F$ function can be decoupled into sectors with different magnetic angular momenta: 


$$
F_{\sigma}(\mathbf{k})=\sum_{m \geq 0} F_{\sigma}^{(m)}\left(k_{\perp}, k_{z}\right) \cos \left(m \phi_{k}+\theta_{m}\right)
$$

where $\theta_{m}$ is an arbitrary phase shift that turns out to be irrelevant to the final solution of $E_{3}$. Note that due to Fermi statistics, the ground state is in the $m=1$ sector. Given $F_{\sigma}(\mathbf{k})$, the wave function $\Psi^{(3)}$ can be obtained accordingly [47].

In Fig. 3, we plot the ground-state trimer energy $E_{3}$ for the ${ }^{40} \mathrm{~K}(\tilde{a})-{ }^{40} \mathrm{~K}(\tilde{a})-{ }^{6} \mathrm{Li}(b)$ case as a function of interaction strength $1 /\left(\lambda a_{s}\right)$. As expected, when $1 /\left(\lambda a_{s}\right)$ increases, the trimer is found to emerge well before the dimer, which leads to the occurrence of the Borromean binding. For the ${ }^{40} \mathrm{~K}-{ }^{40} \mathrm{~K}-{ }^{6} \mathrm{Li}$ system, the Borromean state is stable within the range of $1 /\left(\lambda a_{s}\right) \in[0.2,0.31)$, while the most tightly bound Borromean occurs at the phase boundary against the ordinary trimer, i.e., when the dimer starts to develop at $1 /\left(\lambda a_{s}\right)=0.31$. At this point, the Borromean binding energy can be as large as $\sim 30 \%$ of the SOC energy $\lambda^{2} /\left(2 m_{a}\right)$. The ordinary trimer finally merges into the atom-dimer threshold at a larger $1 /\left(\lambda a_{s}\right)=0.76$.

To gain further understanding of the binding mechanism, we plot in Fig. 4(a) the momentum distribution of the Borromean state at $1 /\left(\lambda a_{s}\right)=0.3$. In contrast to that of dimers shown in Figs. 2(b1) and 2(b2), here, most of the weight of the probability distribution $\left|\Psi^{(3)}(\mathbf{0} ; \mathbf{k},-;-\mathbf{k},-)\right|^{2}$ spreads along the $\mathrm{U}(1)$ circle in the lowest-energy subspace for $\tilde{a}$ atoms. Thus, scattering among these low-energy states contributes the most to the bound-state formation, consistent with the schematics in Fig. 1(c).

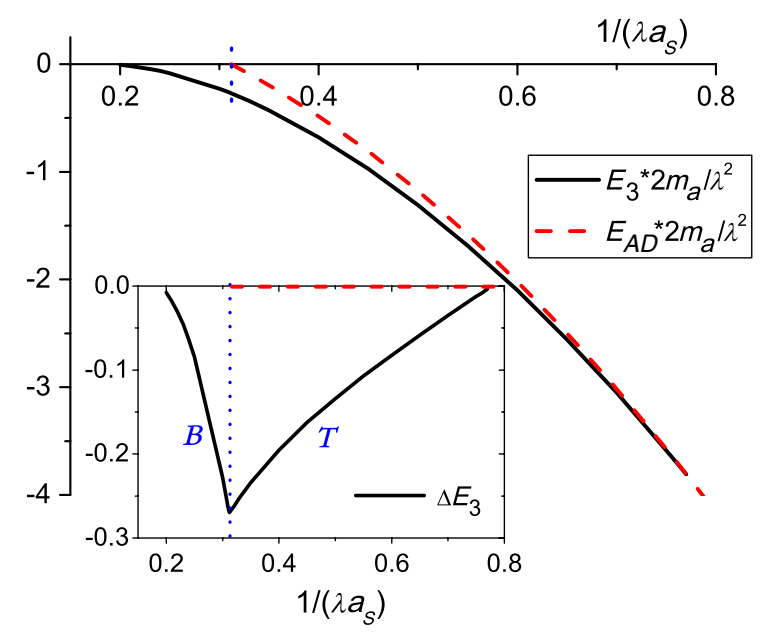

FIG. 3. Borromean binding of the ${ }^{40} \mathrm{~K}(\tilde{a})-{ }^{40} \mathrm{~K}(\tilde{a})-{ }^{6} \mathrm{Li}(b)$ system. The trimer binding energy $E_{3}$ (solid black line) and atomdimer threshold $E_{\mathrm{AD}}$ (dashed red line) are shown as functions of $1 /\left(\lambda a_{s}\right)$. Energies are in units of the SOC energy $\lambda^{2} /\left(2 m_{a}\right)$. The trimer with $E_{3}<0$ and dimer with $E_{2}<0$, respectively, emerge at $1 /\left(\lambda a_{s}\right)=0.2$ and 0.31 . The inset shows $\Delta E_{3}$, the relative value of $E_{3}$ compared to the scattering threshold or the atomdimer threshold. The dotted vertical line marks the boundary between Borromean $(B)$ and ordinary trimer $(T)$ states.

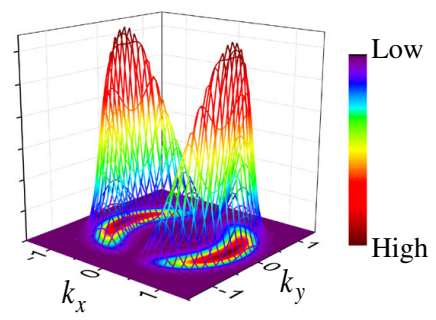

(a)

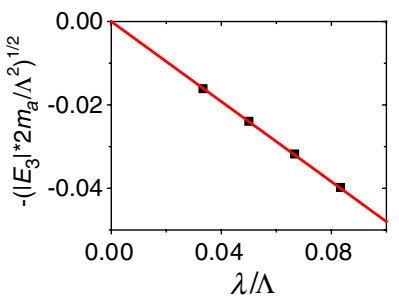

(b)
FIG. 4. Momentum distribution and universality of the Borromean bound state. (a) Probability distribution $\left|\Psi^{(3)}(\mathbf{0} ; \mathbf{k},-;-\mathbf{k},-)\right|^{2}$ for the Borromean binding at $1 /\left(\lambda a_{s}\right)=$ 0.3 and $\eta=40 / 6$. The phase shift $\theta_{m}$ is chosen to be 0 . (b) Borromean binding energy $E_{3}$ [in units of cutoff energy $\left.\Lambda^{2} /\left(2 m_{a}\right)\right]$ as a function of $\lambda / \Lambda$ for four different cutoffs $\Lambda$. Other parameters are the same as in (a).

An outstanding feature of the Borromean binding in the current system is its universality; i.e., the binding energy does not rely on the short-range interaction details. The universality can be shown by imposing different highmomentum cutoffs $\Lambda$ for the argument of the $F_{\sigma}$ function in Eq. (7): $\left(k_{\perp}^{c},\left|k_{z}\right|^{c}\right)=(\sqrt{2} \Lambda, \Lambda)$. In Fig. 4(b), we plot $E_{3}$ as a function of $\lambda / \Lambda$ for the Borromean binding at $1 /\left(\lambda a_{s}\right)=0.3$. If the binding is universal, $E_{3}$ should be independent of the actual cutoff $\Lambda$, and all the points should fall onto a straight line in the $\left\{\lambda / \Lambda, \sqrt{E_{3} /\left[\Lambda^{2} /\left(2 m_{a}\right)\right]}\right\}$ plane, which is exactly the case in Fig. 4(b). The only relevant length scales are then $a_{s}$ and $1 / \lambda$. The universality of the Borromean binding here distinguishes itself from those in the previous studies where the short-range (or high-energy) details of the interaction potential play essential roles.

Furthermore, we find that the Borromean binding in the current system is remarkably robust. As shown in the ground-state phase diagram for the $\tilde{a}-\tilde{a}-b$ system in Fig. 5,

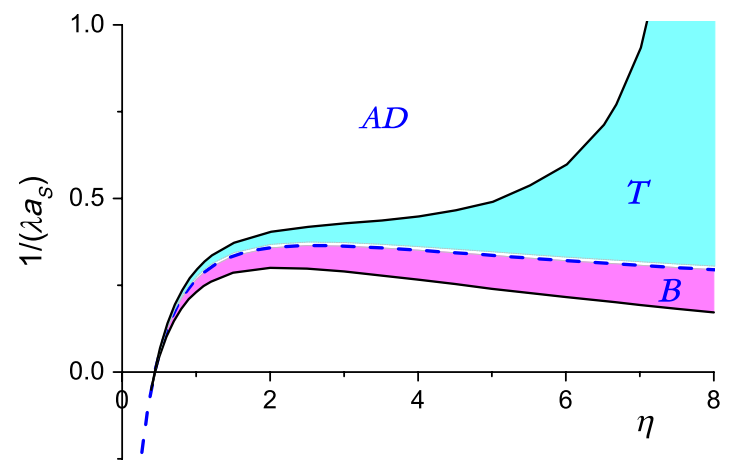

FIG. 5. Phase diagram for trimer states. The boundaries are shown in terms of $1 /\left(\lambda a_{s}\right)$ and $\eta=m_{a} / m_{b}$. The lower and upper solid curves, respectively, show the threshold of Borromean $(B)$ binding and the boundary at which the ordinary trimer $(T)$ merges into the atom-dimer continuum $(A D)$. The dashed blue curve is the dimer threshold [see Fig. 2(a)], which also marks the boundary between $B$ and $T$ for $\eta \geq 0.39$. 
the Borromean binding can be stabilized over a wide range of mass ratios with $\eta \geq 0.39$, thus covering all $\mathrm{Li}-\mathrm{Li}-\mathrm{Li}$, $\mathrm{K}-\mathrm{K}-\mathrm{K}$, and $\mathrm{K}-\mathrm{K}-\mathrm{Li}$ systems. We have checked that the momentum distributions of these Borromean states for different $\eta$ all exhibit similar structures, as shown in Fig. 4(a). Therefore, these Borromean states all share the same binding mechanism, which is closely related to the spectral symmetry in the low-energy manifold due to Rashba SOC (see Fig. 1). This mechanism also ensures the robustness of such a binding against changes in the spin dependence of the interaction. Our results are thus not limited to the spin-selective interaction considered in this work.

\section{FINAL REMARK}

The universal Borromean bindings demonstrated in our work are expected to have dramatic effects on the manybody system. With the Borromean binding energy on the same order of the SOC energy, a dilute gas with strong SOC is anticipated to be comprised of self-bound Borromean clusters that function as composite fermions. Moreover, as the emergence of such a binding is associated with the three-body scattering resonance, a scattering system near this resonance will exhibit strong three-body correlations that dominate over the two-body ones. These prominent three-body correlations would potentially lead to intriguing collective phenomena in both the attractive and the scattering branches of the underlying fermion system.

Finally, we remark that the mechanism of universal Borromean bindings established in this work can be generalized to a vast class of systems, where the singleparticle spectral symmetry is modified by intrinsic or external potentials. Our work thus paves the way for the study of systems where the single-particle physics, instead of interaction details, plays the dominant role in generating exotic few-body correlations, which should lead to new quantum phases in many-body systems.

\section{ACKNOWLEDGMENTS}

This work is supported by the NFRP (No. 2011CB921200 and No. 2011CBA00200), the NNSF (No. 60921091), the NSFC (No. 11104158, No. 11374177, No. 11105134, and No. 11374283), the Fundamental Research Funds for the Central Universities (No. WK2470000006), and the programs of the Chinese Academy of Sciences.

[1] C. Mao, W. Sun, and N. C. Seeman, Assembly of Borromean Rings from DNA, Nature (London) 386, 137 (1997).

[2] K. S. Chichak, S. J. Cantrill, A. R. Pease, S.-H. Chiu, G. W. V. Cave, J. L. Atwood, and J. F. Stoddart, Molecular Borromean Rings, Science 304, 1308 (2004).

[3] M. V. Zhukov, B. V. Danilin, D. V. Fedorov, J. M. Bang, I. S. Thompson, and J.S. Vaagen, Bound State Properties of
Borromean Halo Nuclei: ${ }^{6} \mathrm{He}$ and ${ }^{11} \mathrm{Li}$, Phys. Rep. 231, 151 (1993).

[4] D. V. Fedorov, A. S. Jensen, and K. Riisager, Three-Body Halos: Gross Properties, Phys. Rev. C 49, 201 (1994).

[5] T. Kraemer, M. Mark, P. Waldburger, J. G. Danzl, C. Chin, B. Engeser, A. D. Lange, K. Pilch, A. Jaakkola, H.-C. Nägerl, and R. Grimm, Evidence of Efimov Quantum States in an Ultracold Gas of Caesium Atoms, Nature (London) 440, 315 (2006).

[6] T. B. Ottenstein, T. Lompe, M. Kohnen, A. N. Wenz, and S. Jochim, Collisional Stability of a Three-Component Degenerate Fermi Gas, Phys. Rev. Lett. 101, 203202 (2008).

[7] G. Barontini, C. Weber, F. Rabatti, J. Catani, G. Thalhammer, M. Inguscio, and F. Minardi, Observation of Heteronuclear Atomic Efimov Resonances, Phys. Rev. Lett. 103, 043201 (2009).

[8] M. Zaccanti, B. Deissler, C. D'Errico, M. Fattori, M. Jona-Lasinio, S. Müller, G. Roati, M. Inguscio, and G. Modugno, Observation of an Efimov Spectrum in an Atomic System, Nat. Phys. 5, 586 (2009).

[9] N. Gross, Z. Shotan, S. Kokkelmans, and L. Khaykovich, Observation of Universality in Ultracold ${ }^{7} \mathrm{Li}$ Three-Body Recombination, Phys. Rev. Lett. 103, 163202 (2009).

[10] S. E. Plooack, D. Dries, and R. G. Hulet, Universality in Three-and Four-Body Bound States of Ultracold Atoms, Science 326, 1683 (2009).

[11] S. Nakajima, M. Horikoshi, T. Mukaiyama, P. Naidon, and M. Ueda, Nonuniversal Efimov Atom-Dimer Resonances in a Three-Component Mixture of ${ }^{6} \mathrm{Li}$, Phys. Rev. Lett. 105, 023201 (2010).

[12] M. Berninger, A. Zenesini, B. Huang, W. Harm, H.-C. Nägerl, F. Ferlaino, R. Grimm, P. S. Julienne, and J. M. Hutson, Universality of the Three-Body Parameter for Efimov States in Ultracold Cesium, Phys. Rev. Lett. 107, 120401 (2011).

[13] R. J. Wild, P. Makotyn, J. M. Pino, E. A. Cornell, and D. S. Jin, Measurements of Tan's Contact in an Atomic Bose-Einstein Condensate, Phys. Rev. Lett. 108, 145305 (2012).

[14] R. S. Bloom, M.-G. Hu, T. D. Cumby, and D. S. Jin, Test of Universal Three-Body Physics in an Ultracold Bose-Fermi Mixture, Phys. Rev. Lett. 111, 105301 (2013).

[15] V. Efimov, Weakly-Bound States of Three Resonantly Interacting Particles, Yad. Fiz. 12, 1080 (1970) [Sov. J. Nucl. Phys. 12, 589 (1971)].

[16] E. Braaten and H.-W. Hammer, Universality in Few-Body Systems with Large Scattering Length, Phys. Rep. 428, 259 (2006).

[17] J.-M. Richard and S. Fleck, Limits on the Domain of Coupling Constants for Binding N-Body Systems with No Bound Subsystems, Phys. Rev. Lett. 73, 1464 (1994).

[18] S. Moszkowski, S. Fleck, A. Krikeb, L. Theussl, J. M. Richard, and K. Varga, Binding Three or Four Bosons without Bound Subsystems, Phys. Rev. A 62, 032504 (2000).

[19] E. Nielsen, D. V. Fedorov, and A. S. Jensen, Structure and Occurrence of Three-Body Halos in Two Dimensions, Few-Body Syst. 27, 15 (1999).

[20] A. G. Volosniev, D. V. Fedorov, A. S. Jensen, and N. T. Zinner, Occurrence Conditions for Two-Dimensional Borromean Systems, Eur. Phys. J. D 67, 95 (2013). 
[21] A. G. Volosniev, D. V. Fedorov, A. S. Jensen, and N. T. Zinner, Borromean Ground State of Fermions in Two Dimensions, arXiv:1312.6535.

[22] Y.-J. Lin, K. Jiménez-García, and I. B. Spielman, SpinOrbit-Coupled Bose-Einstein Condensates, Nature (London) 471, 83 (2011).

[23] Y.-J. Lin, R. L. Compton, K. Jiménez-Garcia, W. D. Phillips, J. V. Porto, and I. B. Spielman, A Synthetic Electric Force Acting on Neutral Atoms, Nat. Phys. 7, 531 (2011).

[24] J.-Y. Zhang, S.-C. Ji, Z. Chen, L. Zhang, Z.-D. Du, B. Yan, G.-S. Pan, B. Zhao, Y.-J. Deng, H. Zhai, S. Chen, and J.-W. Pan, Collective Dipole Oscillations of a Spin-Orbit Coupled Bose-Einstein Condensate, Phys. Rev. Lett. 109, 115301 (2012).

[25] R. A. Williams, L. J. LeBlanc, K. Jiménez-Garci, M. C. Beeler, A. R. Perry, W. D. Phillips, and I. B. Spielman, Synthetic Partial Waves in Ultracold Atomic Collisions, Science 335, 314 (2012).

[26] P. Wang, Z.-Q. Yu, Z. Fu, J. Miao, L. Huang, S. Chai, H. Zhai, and J. Zhang, Spin-Orbit Coupled Degenerate Fermi Gases, Phys. Rev. Lett. 109, 095301 (2012).

[27] L. W. Cheuk, A. T. Sommer, Z. Hadzibabic, T. Yefsah, W. S. Bakr, and M. W. Zwierlein, Spin-Injection Spectroscopy of a Spin-Orbit Coupled Fermi Gas, Phys. Rev. Lett. 109, 095302 (2012).

[28] C. Qu, C. Hamner, M. Gong, C. Zhang, and P. Engels, Observation of Zitterbewegung in a Spin-Orbit-Coupled Bose-Einstein Condensate, Phys. Rev. A 88, 021604(R) (2013).

[29] M. C. Beeler, R. A. Williams, K. Jiménez-Garcia, L. J. LeBlanc, A. R. Perry, and I. B. Spielman, The Spin Hall Effect in a Quantum Gas, Nature (London) 498, 201 (2013).

[30] S.-C. Ji, J.-Y Zhang, L. Zhang, Z.-D. Du, W. Zheng, Y.-J. Deng, H. Zhai, S. Chen, and J.-W. Pan, Experimental Determination of the Finite-Temperature Phase Diagram of a Spin-Orbit Coupled Bose Gas, Nat. Phys. 10, 314 (2014).

[31] R. A. Williams, M. C. Beeler, L. J. LeBlanc, K. JimenezGarcia, and I. B. Spielman, Raman-Induced Interactions in a Single-Component Fermi Gas Near an s-Wave Feshbach Resonance, Phys. Rev. Lett. 111, 095301 (2013).

[32] Z. Fu, L. Huang, Z. Meng, P. Wang, L. Zhang, S. Zhang, H. Zhai, P. Zhang, and J. Zhang, Production of Feshbach Molecules Induced by Spin-Orbit Coupling in Fermi Gases, Nat. Phys. 10, 110 (2014).

[33] D. L. Campbell, G. Juzeliūnas, and I. B. Spielman, Realistic Rashba and Dresselhaus Spin-Orbit Coupling for Neutral Atoms, Phys. Rev. A 84, 025602 (2011).
[34] J. D. Sau, R. Sensarma, S. Powell, I. B. Spielman, and S. Das Sarma, Chiral Rashba Spin Textures in Ultracold Fermi Gases, Phys. Rev. B 83, 140510(R) (2011).

[35] Z. F. Xu and L. You, Dynamical Generation of Arbitrary Spin-Orbit Couplings for Neutral Atoms, Phys. Rev. A 85, 043605 (2012).

[36] X.-J. Liu, K. T. Law, and T. K. Ng, Realization of $2 D$ Spin-Orbit Interaction and Exotic Topological Orders in Cold Atoms, Phys. Rev. Lett. 112, 086401 (2014).

[37] B. M. Anderson, I. B. Spielman, and G. Juzeliūnas, Magnetically Generated Spin-Orbit Coupling for Ultracold Atoms, Phys. Rev. Lett. 111, 125301 (2013).

[38] Z.-F. Xu, L. You, and M. Ueda, Atomic Spin-Orbit Coupling Synthesized with Magnetic-Field-Gradient Pulses, Phys. Rev. A 87, 063634 (2013).

[39] B. M. Anderson, G. Juzeliūnas, V. M. Galitski, and I. B. Spielman, Synthetic 3D Spin-Orbit Coupling, Phys. Rev. Lett. 108, 235301 (2012).

[40] H. Zhai, Spin-Orbit Coupled Quantum Gases, Int. J. Mod. Phys. B 26, 1230001 (2012); Degenerate Quantum Gases with Spin-Orbit Coupling, arXiv:1403.8021; V. Galitski and I. B. Spielman, Spin-Orbit Coupling in Quantum Gases, Nature (London) 494, 49 (2013); N. Goldman, G. Juzeliūnas, P. Öhberg, and I. B. Spielman, Light-Induced Gauge Fields for Ultracold Atoms, arXiv:1308.6533; X. Zhou, Y. Li, Z. Cai, and C. Wu, Unconventional States of Bosons with the Synthetic Spin-Orbit Coupling, J. Phys. B 46, 134001 (2013).

[41] J. P. Vyasanakere and V. B. Shenoy, Bound States of Two Spin- $\frac{1}{2}$ Fermions in a Synthetic Non-Abelian Gauge Field, Phys. Rev. B 83, 094515 (2011).

[42] X. Cui, Mixed-Partial-Wave Scattering with Spin-Orbit Coupling and Validity of Pseudopotentials, Phys. Rev. A 85, 022705 (2012).

[43] Y. Wu and Z. Yu, Short-Range Asymptotic Behavior of the Wave Functions of Interacting Spin- $\frac{1}{2}$ Fermionic Atoms with Spin-Orbit Coupling: A Model Study, Phys. Rev. A 87, 032703 (2013).

[44] Z. Y. Shi, X. Cui, and H. Zhai, Universal Trimers Induced by Spin-Orbit Coupling in Ultracold Fermi Gases, Phys. Rev. Lett. 112, 013201 (2014).

[45] O. I. Kartavtsev and A. V. Malykh, Low-Energy Three-Body Dynamics in Binary Quantum Gases, J. Phys. B 40, 1429 (2007).

[46] C. Chin, R. Grimm, P. Julienne, and E. Tiesinga, Feshbach Resonances in Ultracold Gases, Rev. Mod. Phys. 82, 1225 (2010).

[47] See Supplemental Material at http://link.aps.org/ supplemental/10.1103/PhysRevX.4.031026 for the details in deriving and solving two-body and three-body equations. 